\title{
Practical Considerations for Electron Holography on Doped Semiconductor Devices
}

\author{
Alexander Thesen ${ }^{*}$, Bernhard G. Frost ${ }^{*}$, David C. Joy ${ }^{*}$ \\ *EM Facility, University of Tennessee, Knoxville, TN 37996 and High Temperatures Materials \\ Laboratory, Oak Ridge National Laboratory, Bethel Valley Road 1, Oak Ridge, TN 37831
}

Recently a lot of attention has been given to off-axis transmission electron holography as a means for performing 2 dimensional dopant profiling of CMOS devices [1-3]. So far, however, a quantitative interpretation of the data has been a challenge. This paper discusses the problems, which make the precise quantitative analysis of the phase image difficult and shows possible ways to solve or avoid them.

The Sample:

In order to maximize the resolution of the phase shift caused by the voltage trop over the junction an optimum sample thickness can be determined to be approximately twice the inelastic mean free path. Assuming a value for the inelastic mean free path in silicon of about $95 \mathrm{~nm}$ at $200 \mathrm{kV}$ the opimum sample thickness computes to $190 \mathrm{~nm}$. When preparing the sample with a focused ion beam system great care must be given in order not to amorphize the sample, which decreases doping contrast dramatically. In addition to this the metal deposited during the FIB sample preparation process can charge up in the electron beam overlaying a logarithmic voltage profile. It can also be beneficial to coat the sample, whereas plasma cleaning has been shown to reduce doping contrast.

Imaging:

For a quantitative analysis Fresnel fringes by the biprism overlaying the phase image can be dis turbing. It is beneficial to this end to align the biprism fiber at some angle to the features of interest. This will make it possible to filter out Fresnel fringes in Fourier space without obscuring data.

Interpretation of the results:

When trying to obtain quantitative results the main problem remains how to correct the phase image for phase changes due to thickness variation. In the method used by GajdardziskaJosifovska the amplitude image can be used to generate a thickness map [4]. This method, however, depends on the exact knowledge of inner potential and inelastic mean free path. Both constants are not known to the necessary accuracy and depend on sample tilt and other experimental considerations. We propose to expand the given method by combining both constants into one and varying it until an area of the sample known to be free of doping and charging subtracted from the thickness map shows a minimum variance. The resulting constant can then be used to correct the image.

[1] Rau et al.: Phys. Rev. Lett. 82 (1999), p. 2614

[2] Wang et al.: Appl. Phys. Lett. 80 (2002), p. 246

[3] Frabboni et al.: Ultramicroscopy 23 (1987), p. 29

[4] Gajdardziska-Josifovska and McCartney: Ultramicroscopy 53 (1994), p. 291

[5] This research was supported as part of the user program sponsored by the U.S. Department of Energy under contract DE-AC05-00OR22725 with the Oak Ridge National Laboratory, managed by UT-Battelle, LLC and International SEMATECH 

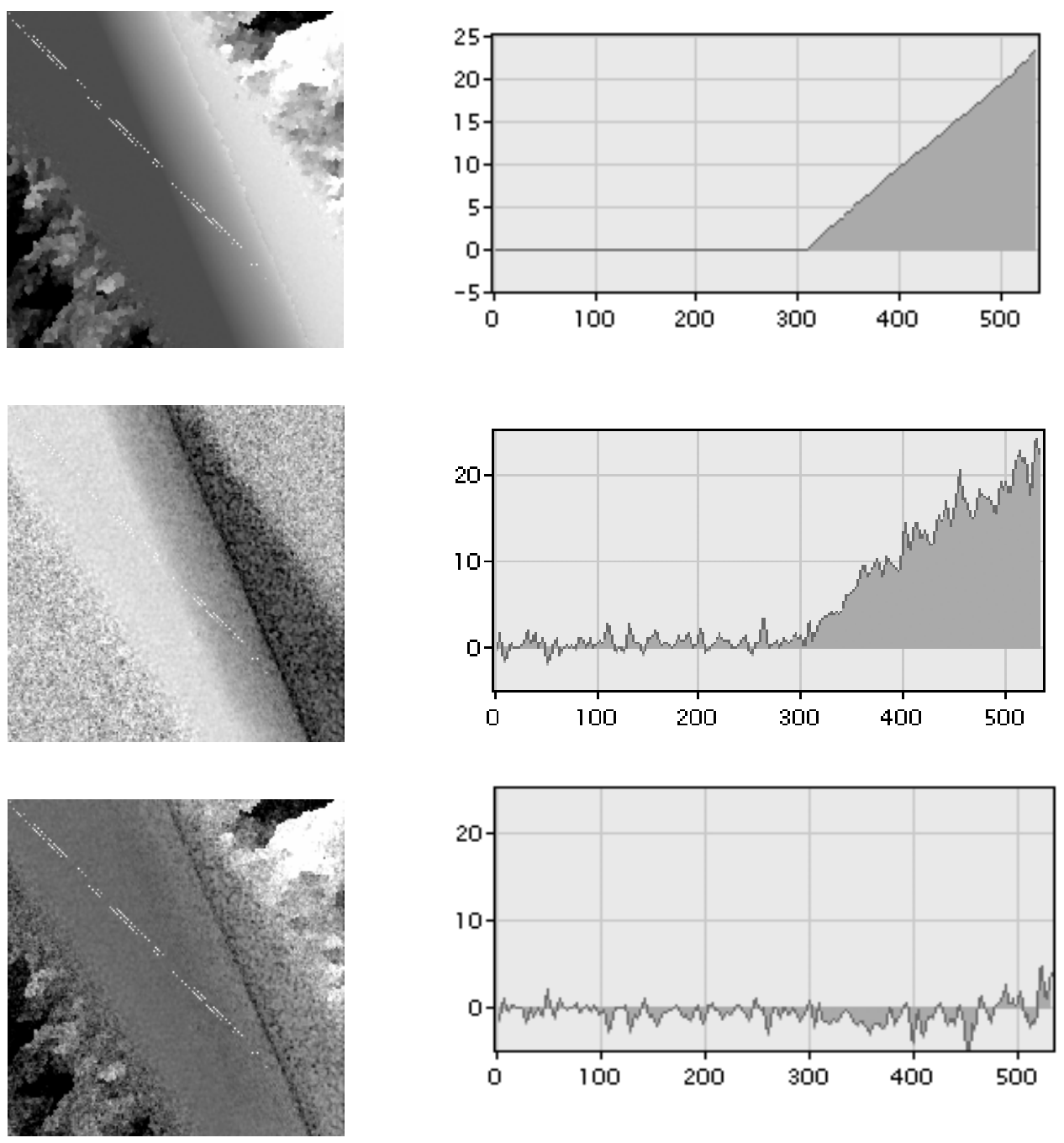

FIG. 1: Elimination of thickness contribution of phase using amplitude information (top: pure phase image, middle: logarithm of amplitude image, bottom: phase image after thickness elimination)

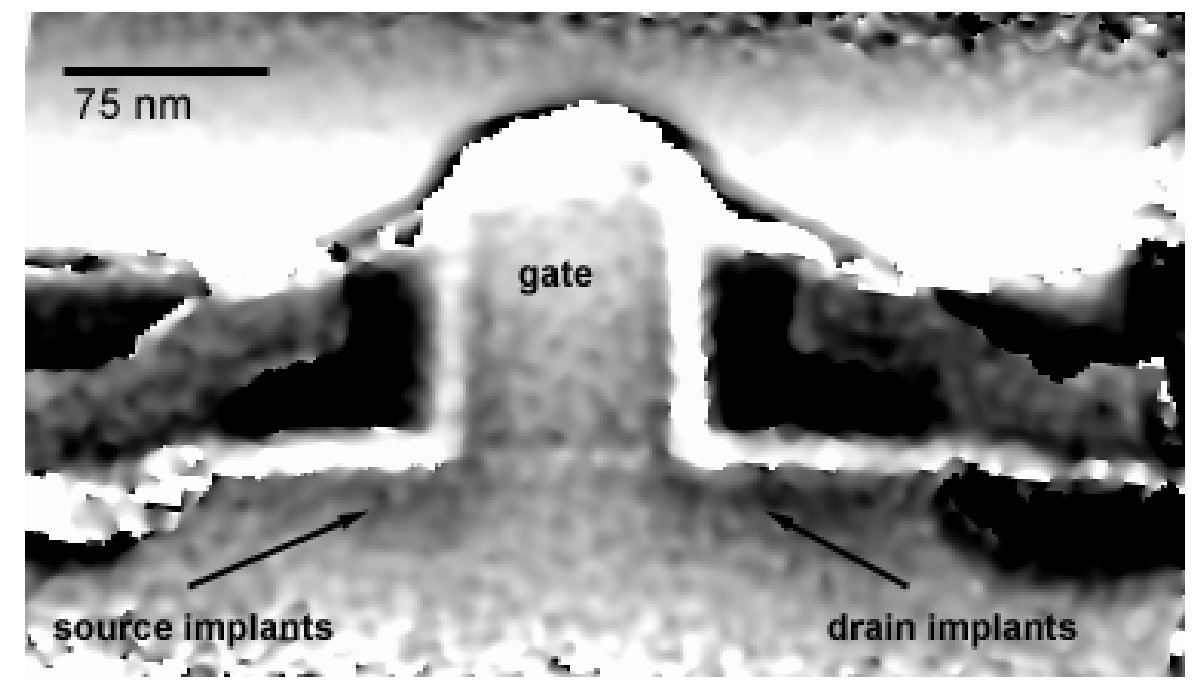

FIG. 2: Phase image of a transistor inside a commercially available microprocessor showing dopant contrast. 